Michael Klein ${ }^{1}$ Claudia Quinten²

\section{Zur Langzeitentwicklung von Kindern stationär behandelter alkoholabhängiger Eltern}

Long Term Development of Children of Treated Alcohol Dependent Parents

\section{Zusammenfassung}

Anliegen: Kinder in suchtbelasteten Familien weisen ein bis sechsfach erhöhtes Risiko auf, selbst abhängigkeitskrank zu werden, und zeigen darüber hinaus mit erhöhter Häufigkeit psychopathologische Symptome. Es wird untersucht, wie sich die Entwöhnungsbehandlung eines Elternteils auf die Langzeitentwicklung der Kinder auswirkt. Methode: Es wurden 98 junge erwachsene Kinder aus suchtbelasteten Familien (Durchschnittsalter 22 Jahre), deren suchtkranker Elternteil vor durchschnittlich 8 Jahren eine stationäre Entwöhnungsbehandlung absolviert hat, umfassend schriftlich befragt. Ergebnisse: Etwa ein Viertel der Probanden haben - zumindest zeitweise - eine Trennung der Eltern erlebt und sind seit ihrer Kindheit schon einmal psychologisch oder psychiatrisch behandelt worden. Eine ähnliche Quote zeigt sich bei der aktuellen Problembelastung, bei der massive psychische Probleme (z.B. Ängste, Depressionen, Zwänge, problematischer Alkoholkonsum) von bis zu 25\% dieser Gruppe angegeben werden. Die Atmosphäre in der Herkunftsfamilie wird von durchschnittlich mehr als 40\% der Probanden als negativ (z.B. disharmonisch, verschlossen, hemmend, instabil, angespannt) beurteilt. Innerhalb der Gruppe der jungen Erwachsenen aus suchtbelasteten Familien stellen im Rahmen dieser Untersuchung diejenigen, die mit zwei suchtkranken Elternteilen oder mit einem Elternteil, welches keine dauerhafte Abstinenz erreichte, aufwuchsen, die beiden Hauptproblemgruppen dar. Es ist von einem hohen Ausmaß intrafamilialer Konflikte, die sich insbesondere im emotionalen Bereich abspielten, auszugehen. Bei der Bewertung der Kindheit bzw. Jugend durch die jungen erwachsenen Probanden stechen die Merkmale Instabilität und Unsicherheit hervor. Schlussfolgerungen: Kinder alkoholabhängiger Eltern leiden unter vielfälti-

\section{Abstract}

Children in addicted families show an elevated, up to sixfold, risk to become addicted themselves during their life time. In addition, from early childhood they have an increased risk for psychopathological and behavioral symptoms. It is investigated whether the treatment of the addicted parent has long term effects on the children's development. 98 young adults from addicted families with an addicted parent who has completed an inpatient treatment program for alcoholism (on the average: 8 years ago) have been investigated intensively. About one quarter of all compilers have experienced - at least temporarily - separation of their parents and have been treated psychologically or psychiatrically at least once. A similar number is to be found concerning those who report to have been feeling strained in their everyday life by symptoms like depression, anxiety etc. The atmosphere in their family of origin has been negatively experienced by more than $40 \%$ of the subjects reporting above all discordant, stressed, unstable, reserved, and inhibiting conditions. Within the group of the young adults from addicted parents those are the most disturbed ones who had experienced both parents being addicted or those whose parents did not stop drinking after therapy. All in all, there has been a high amount of intrafamilial conflicts reported by a great number of subjects. Under preventive perspective it seems appropriate to intervene with COAs as early as possible in order to facilitate better developmental pathways and to prevent pathologies. One possible intervention that can be highly effective is the early inpatient treatment of the addicted parent that results in long term abstinence. 
gen Symptomen. Da diese umso stärker sind, wenn die Eltern keine dauerhafte Abstinenz erreichen, ist die frühzeitige erfolgreiche Behandlung des suchtkranken Elternteils eine wirksame Präventionsmethode. Insgesamt sollten möglichst früh umfassende Hilfen bereitgestellt werden, um eine optimale Entwicklung wahrscheinlicher zu machen bzw. erste auftretende Störungen schnell zu behandeln.

\section{Schliuisselwörter}

Kinder von Alkoholabhängigen · Langzeitentwicklung · Transmission psychischer Störungen · Suchtrisiken · Suchtfamilie
Key words

Children of alcoholics · long term development · transmission of psychological dysfunctions · risks of addiction · addictive families

\section{Einführung}

Dass Alkoholabhängige überhaupt Kinder haben, wurde lange Zeit von der Suchtforschung und Suchthilfe ignoriert, obwohl die Zahl der betroffenen Kinder insgesamt sehr hoch ist. In Deutschland ist nach offiziellen Angaben von etwa 2 Millionen Alkoholabhängigen auszugehen. Demnach dürften bis zu 2 Millionen Kinder durch die Abhängigkeit eines Elternteils betroffen sein. Als erwachsene Kinder aus alkoholbelasteten Familien sind weitere 5-6 Millionen Personen potenziell durch eine erhöhte Vulnerabilität gegenüber psychischen Störungen gefährdet [1].

In neueren repräsentativen epidemiologischen Studien [2] bestätigte sich, dass die familiäre Belastung durch elterliche Suchterkrankungen hoch ist. Für eine Stichprobe von 3021 Jugendlichen und Jungerwachsenen im Alter zwischen 14 und 24 Jahren stellte sich eine Quote elterlicher Alkoholabhängigkeit bzw. elterlichen Alkoholmissbrauchs von 15,1\% heraus.

Die vorliegende Untersuchung beschäftigt sich mit den Belastungen und der Langzeitentwicklung von Kindern aus Familien, von denen ein suchtkrankes Elternteil eine stationäre Entwöhnungsbehandlung absolviert hat. Dabei waren diese Kinder im Rahmen des psychotherapeutischen Konzepts der Klinik für drei Tage an der stationären Behandlung in Form so genannter Kinder- und Jugendseminare beteiligt.

\section{Kinder von Alkoholikern als Thema in Forschung und Praxis}

In einer Vielzahl von Studien wurde nachgewiesen [3-5], dass Alkoholabhängige überzufällig oft aus Familien stammen, in denen bereits ein Elternteil oder beide abhängig waren. Im Rahmen einer umfangreichen Untersuchung [6] zeigte sich, dass von knapp 4000 alkoholabhängigen Personen 30,8\% einen abhängigen Elternteil aufwiesen. Demgegenüber gaben in den nichtklinischen Kontrollstichproben von 922 Personen lediglich 4,7\% einen abhängigen Elternteil an.

Eine Langzeitstudie über einen Zeitraum von 33 Jahren [7] brachte für erwachsene Kinder aus Suchtfamilien in 28\% der Fälle eine Diagnose für Alkoholabhängigkeit. Männer mit einem abhängigen Vater hatten mehr als doppelt so häufig eine Alkoholabhängigkeit als Männer ohne abhängigen Vater [vgl. 8-10].
Diese Studien belegen zusammen mit einer Vielzahl anderer Untersuchungen (siehe zusammenfassend: [11,2]), dass Kinder von Alkoholikern, und zwar vor allem Söhne, als Risikogruppe für die Entwicklung von Alkoholmissbrauch und -abhängigkeit angesehen werden müssen. Insgesamt kann davon ausgegangen werden, dass diese Kinder ein bis zu sechsfach höheres Risiko haben, selber abhängig zu werden oder Alkohol zu missbrauchen. Ausreichend belegt ist aber auch, dass für Kinder in suchtbelasteten Familien das Risiko der Erkrankung an anderen psychischen Störungen (insbesondere Angststörungen, Depressionen, antisoziale und hyperkinetische Störungen, Persönlichkeitsstörungen) deutlich - wenn auch nicht so stark wie für Abhängigkeitserkrankungen - erhöht ist [12].

Jedoch ist ausdrücklich nicht davon auszugehen, dass alle Kinder von Alkoholikern eine eigene Abhängigkeit oder andere psychische Störungen entwickeln müssen. Vielmehr spielen offenbar zahlreiche Faktoren bei der Transmission von Störungen, also der Weitergabe einer Krankheit von der Elterngeneration auf die Kinder, eine wichtige Rolle. Einzelne Moderatorvariablen vermögen das Risiko in einer verstärkenden oder abschwächenden Form zu beeinflussen.

\section{Methodik}

Zur Bildung einer Untersuchungsgruppe von Kindern aus suchtbelasteten Familien wurden alle Teilnehmerinnen und Teilnehmer der Kinder- und Jugendseminare der Fachklinik Thommener Höhe (heute: Kliniken Daun, Darscheid) zwischen 1984 und 1995 herangezogen. Von diesen 551 Personen wurden nur solche ausgewählt, die zum Zeitpunkt der Befragung mindestens 18 Jahre alt waren. Dies geschah vor dem Hintergrund, dass diese Personen inzwischen volljährig sein sollten, damit zur Beantwortung des Fragebogens nicht das Einverständnis der Eltern eingeholt werden musste. Was jedoch noch wichtiger erschien, war die Vorgabe, dass die Teilnahme am Kinder- oder Jugendseminar einige Zeit zurückliegen musste. Als Mindestkriterium hierfür wurden 2 Jahre angenommen, da das Höchstalter zur Teilnahme am Kinder- und Jugendseminar bei 16 Jahren liegt. Aus diesen Kriterien ergab sich eine Ausgangsgruppe von 219 jungen Erwachsenen.

Für diese 219 Personen erfolgte eine Adressermittlung über die der Klinik vorliegende letzte bekannte Elternadresse. Diese Strategie führte nach verschiedenen weiteren intensiven Bemühun- 
gen (z. B. über die Telefonauskunft) zu einer Ausgangsgruppe von 194 jungen erwachsenen Kindern der ehemaligen Patienten, die auf der Grundlage einer vorliegenden Adresse mit einem ausführlichen Befragungsinstrument angeschrieben wurden.

Alle hatten mindestens einen Elternteil, der in der genannten Fachklinik wegen seiner Abhängigkeitserkrankung behandelt worden war. Von den 194 angeschriebenen Personen waren 98 (50,5\%) männlichen, 96 (49,5\%) weiblichen Geschlechts.

\section{Untersuchungsdurchführung}

Die im vorausgehenden Abschnitt geschilderte Herleitung der Untersuchungsgruppe bildete die Basis für die schriftliche Befragung der ehemaligen Seminarteilnehmerinnen und -teilnehmer nach ihren familien- und lebensgeschichtlichen Erfahrungen im Kontext suchtbelasteter Familien. Die 194 genannten Personen erhielten einen Fragebogen in einem neutralen Umschlag mit einem erklärenden Anschreiben und einem Freiumschlag zur Rücksendung.

Nach jeweils zwei Wochen bekamen die Untersuchungsteilnehmer ein Erinnerungsschreiben mit der freundlichen Bitte zugeschickt, den zugesandten Fragebogen möglichst bald ausgefüllt zurückzusenden. So weit als möglich wurden zusätzlich Telefongespräche mit den Betroffenen geführt, um die Compliance zur Mitwirkung an der Untersuchung weiter zu erhöhen. Diese Bemühungen führten schließlich zu einem Rücklauf ausgefüllter Fragebogen von $n=98$ (50,5\%) innerhalb von 6 Monaten nach Versendung des ersten Anschreibens.

\section{Ergebnisse}

\section{Antwortverhalten}

Die genannte Rücklaufquote ausgefüllter Fragebogen von 50,5\% wurde zunächst auf Geschlechtseffekte hin untersucht (Tab.1).

Tab. 1 Antwortverhalten in Abhängigkeit vom Geschlecht (Häufigkeiten und Prozentwerte [in Klammern])

\begin{tabular}{lllc}
\hline Kategorie & männlich & weiblich & gesamt \\
\hline Angeschriebene & $98(50,5)$ & $96(49,5)$ & 194 \\
\hline Antworter & $38(38,8)$ & $60(61,2)$ & 98 \\
\hline
\end{tabular}

Es zeigt sich dabei, dass von den 98 männlichen Probanden der Ausgangsgruppe lediglich 38 (38,8\%) geantwortet haben. Für die weiblichen Mitglieder der Untersuchungsgruppe ist das Verhältnis wesentlich günstiger: Hier haben von 96 Angeschriebenen 60 (62,5\%) geantwortet.

Dieser statistisch nur schwach signifikante Unterschied im Antwortverhalten (Chi-Quadrat $=3,60 ; p<0,10$ ) mag zum einen auf generelle geschlechtsspezifische Differenzen in der Mitwirkungsbereitschaft bei derartigen Befragungen beruhen. Zum an- deren könnten weitere Gründe ursächlich wichtig sein, wie z.B. die Möglichkeit, dass die Männer der Untersuchungsgruppe in einem viel höheren Ausmaß als die Frauen schon von psychischen, insbesondere suchtbedingten Störungen betroffen waren, aufgrund derer sie nicht bereit oder in der Lage waren, zu antworten. Auch die Bereitschaft, sich kritisch mit der eigenen Familiengeschichte und der Rolle der Eltern auseinander zu setzen, mag geschlechtsspezifisch unterschiedlich sein.

\section{Soziodemografische Daten und zentrale lebensgeschichtliche Ereignisse}

Die Auswertung der Fragebogendaten fokussierte zunächst auf soziodemografische Daten bezüglich der Kindheit und Jugend der Befragten:

Als Einzelkinder wuchsen 17 (17,3\%), als Älteste in einer Geschwisterfolge 43 (43,9\%), als Mittlere 9 (9,2\%) und als Jüngste 29 (29,6\%) auf. Sechs der Probanden sind aufgrund von Trennungen bzw. Scheidungen der Eltern mit Halb- oder Stiefgeschwistern aufgewachsen.

Zum Zeitpunkt des Kinder- bzw. Jugendseminars betrug das Durchschnittsalter der Probanden 13,0 Jahre (Std. abw.: 3,12 Jahre). Die durchschnittliche Katamnesedauer, d.h. die Dauer vom Zeitpunkt des Seminars in der Fachklinik bis zur vorliegenden Erhebung, beläuft sich auf 7,66 Jahre (Std. abw.: 2,90 Jahre).

Neben diesen soziodemografischen Daten aus der Kindheit und Jugend der Befragten interessierten zentrale lebensgeschichtliche Erfahrungen und Erlebnisse der jungen Erwachsenen. So berichten $6(6,2 \%)$, dass sie schon einmal in einem Heim untergebracht waren, 7 (7,1\%) lebten - zumindest zeitweise - bei Pflegeeltern. Von allen Antwortern haben 27 (27,5\%) eine Trennung der Eltern erlebt. Für 15 (55,5\%) von diesen bedeutete dies nach eigener Einschätzung ein negatives Ereignis. 67 (68\%) berichten von Schulproblemen. Von den 98 Antwortern sind 26 (26,8\%) schon einmal psychologisch oder psychiatrisch behandelt worden. $9(34,6 \%)$ von diesen bewerten dies negativ.

\section{Atmosphäre in der Herkunftsfamilie}

Bezüglich der erlebten Atmosphäre (Tab. 2) berichten zwischen 14,6\% und 56,1\% der Probanden von problematischen Erfahrungen. Je nach Beurteilungsbereich schwankt dieser Wert: Eine gewalttätige Familienatmosphäre berichtet etwa jeder Siebte (14,6\%), eine unfreundliche bzw. kalte etwa jeder Vierte (25\% bzw. 28,9\%), eine unberechenbare bzw. verlogene Atmosphäre haben $35,2 \%$ bzw. $45,4 \%$ erlebt. Noch höhere Werte ergeben sich für die meisten weiteren Merkmale: bedrückt $(41,7 \%)$, disharmonisch (41,8\%), hemmend (42,3\%), verlogen ( $45,4 \%)$, instabil $(54,7 \%)$ und angespannt (56,1\%). Auffällig ist dabei besonders, dass die Indikatoren für mangelnde Stabilität und erhöhten intrafamilialen Stress die jeweils höchsten Werte erreichten.

Bei einer Differenzierung der Antworten nach Subgruppen (Probanden mit suchtkrankem Vater, mit suchtkranker Mutter, mit zwei suchtkranken Elternteilen und mit einem Elternteil, der nach der Therapie keine dauerhafte Abstinenz erreichte) zeigen sich erhebliche weitere Unterschiede. 
Tab. 2 Atmosphäre in der Herkunftsfamilie (alle Angaben in Prozent)

\begin{tabular}{|c|c|c|c|c|c|}
\hline Kategorie & $\begin{array}{l}\text { alle } \\
n=98\end{array}$ & $\begin{array}{l}\text { mit } \\
\text { suchtkrankem } \\
\text { Vater } \\
n=61\end{array}$ & $\begin{array}{l}\text { mit } \\
\text { suchtkranker } \\
\text { Mutter } \\
n=24\end{array}$ & $\begin{array}{l}\text { mit zwei } \\
\text { suchtkranken } \\
\text { Elternteilen } \\
n=13\end{array}$ & $\begin{array}{l}\text { mit Eltern } \\
\text { ohne dauerhafte } \\
\text { Abstinenz } \\
n=26\end{array}$ \\
\hline disharmonisch & 41,8 & 41,8 & 38,1 & 54,5 & 50 \\
\hline kalt & 28,9 & 25,9 & 28,6 & 54,5 & 23,1 \\
\hline verschlossen & 42,7 & 43,6 & 42,9 & 33,7 & 60 \\
\hline unberechenbar & 35,2 & 33,1 & 33,7 & 22,2 & 54,2 \\
\hline gewalttätig & 14,6 & 16,7 & 0 & 30 & 20 \\
\hline verlogen & 45,4 & 43,6 & 47,6 & 50 & 57,3 \\
\hline bedrückt & 41,7 & 40,7 & 42,9 & 50 & 40 \\
\hline hemmend & 42,3 & 43,6 & 42,9 & 40 & 57,7 \\
\hline unfreundlich & 25 & 23,6 & 19 & 44,4 & 42,3 \\
\hline instabil & 54,7 & 51,9 & 57,1 & 55,6 & 72 \\
\hline angespannt & 56,1 & 52,7 & 61,9 & 54,5 & 76,9 \\
\hline
\end{tabular}

Tab. 3 Körperliche Gewalt in der Herkunftsfamilie (alle Angaben in Prozent)

\begin{tabular}{|c|c|c|c|c|c|}
\hline Kategorie & $\begin{array}{l}\text { alle } \\
n=98\end{array}$ & $\begin{array}{l}\text { mit } \\
\text { suchtkrankem } \\
\text { Vater } \\
n=61\end{array}$ & $\begin{array}{l}\text { mit } \\
\text { suchtkranker } \\
\text { Mutter } \\
n=24\end{array}$ & $\begin{array}{l}\text { mit zwei } \\
\text { suchtkranken } \\
\text { Elternteilen } \\
n=13\end{array}$ & $\begin{array}{l}\text { mit Eltern } \\
\text { ohne dauerhafte } \\
\text { Abstinenz } \\
n=26\end{array}$ \\
\hline täglich & 3,1 & 5,5 & 0 & 0 & 3,8 \\
\hline \multicolumn{6}{|c|}{ oder fast täglich } \\
\hline oft & 6,1 & 9,1 & 0 & 9,1 & 3,8 \\
\hline \multicolumn{6}{|c|}{ (mehr als 5x monatl.) } \\
\hline manchmal & 4,1 & 3,6 & 4,8 & 9,1 & 3,8 \\
\hline \multicolumn{6}{|c|}{ (bis zu $5 x$ monatlich) } \\
\hline selten & 36,7 & 40 & 23,8 & 36,3 & 50 \\
\hline \multicolumn{6}{|c|}{ (höchstens 1x im Monat) } \\
\hline nie & 50 & 41,8 & 71,4 & 45,5 & 38,6 \\
\hline
\end{tabular}

Während die Probanden mit ausschließlich suchtkrankem Vater ( $n=61$ ) im Wesentlichen um oder leicht unter den Prozentwerten der Gesamtgruppe liegen, zeigen sich für die Probanden mit suchtkranker Mutter $(n=24)$ bei einigen Werten stärkere Abweichungen. So berichten diese Jungerwachsenen von einer weniger gewalttätigen ( $0 \%$ bei suchtkranken Müttern im Vergleich zu 16,7\% bei suchtkranken Vätern) und weniger unfreundlichen (19\% im Vergleich zu 23,6\%) Familienatmosphäre. Andererseits erlebten sie in höherem Maße Instabilität (57,1\% im Vergleich zu 51,9\%) und Anspannung (61,9\% im Vergleich zu 52,7\%). Erstaunlich ist auch der differenzielle Einfluss der mütterlichen Abhängigkeit auf die Familienatmosphäre: Weniger interaktioneller Stress durch Gewalt, Aggressivität und Unfreundlichkeit bei höherem intrapsychischem Stress durch Anspannungen und Instabilität herrschen hier vor.

Ein wesentlich klareres Bild bezüglich dysfunktionaler und pathogener Bedingungen in suchtbelasteten Familien ergibt sich bei Berücksichtigung der beiden letzten Subgruppen: Dies sind Probanden, von denen beide Elternteile suchtkrank waren
( $n=13$ ) oder bei denen das suchtkranke Elternteil nach der Therapie in der Fachklinik keine dauerhafte Abstinenz erreichte $(\mathrm{n}=26)$. Wenn beide Elternteile suchtkrank sind, wird die Familienatmosphäre etwa doppelt so häufig als kalt (54,5\% im Vergleich zu 28,9\%), gewalttätig (30\% im Vergleich zu 14,6\%) und unfreundlich (44,4\% im Vergleich zu 25\%) eingeschätzt. Eine weniger deutliche Steigerung ergibt sich für die atmosphärischen Variablen „disharmonisch“, „verlogen“ und „bedrückt“. Interessanterweise zeigen sich die Variablen „verschlossen“ und „unberechenbar“ im Vergleich zur Gesamtgruppe erniedrigt, was sollte es angesichts der relativ kleinen Stichprobe kein Zufallseffekt sein - an den speziellen Bedingungen der Suchtbelastung beider Elternteile liegen könnte. Möglicherweise sind die Abläufe in diesen Familien, auch wenn sie negativ erscheinen, für die Kinder berechenbarer und offener als in Familien mit nur einem alkoholabhängigen Elternteil. Die klinische Erfahrung jedenfalls unterstützt eine derartige Hypothese eher. 
Einschätzung der Kindheit

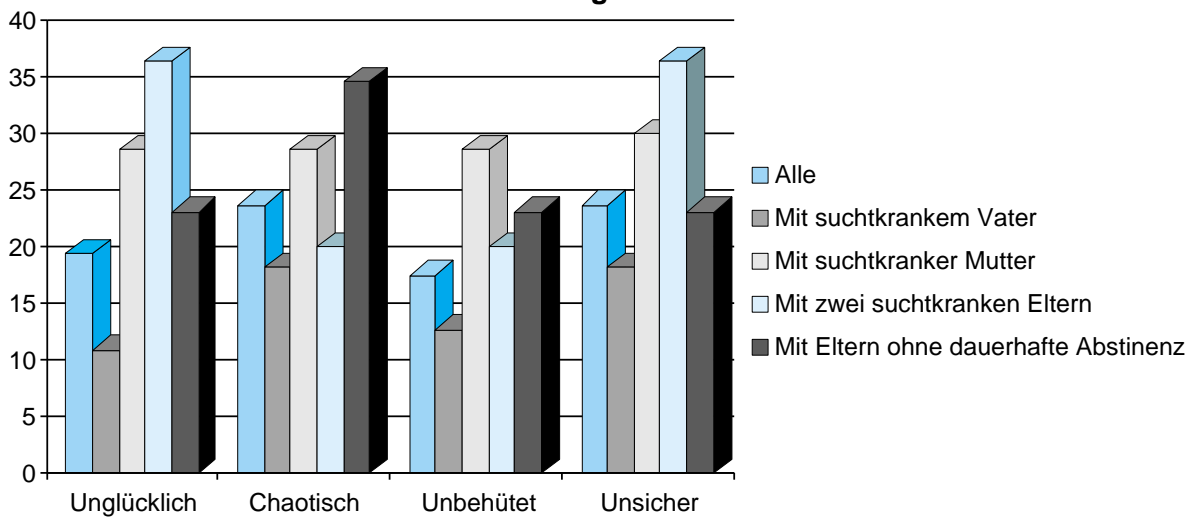

Einschätzung der Jugend

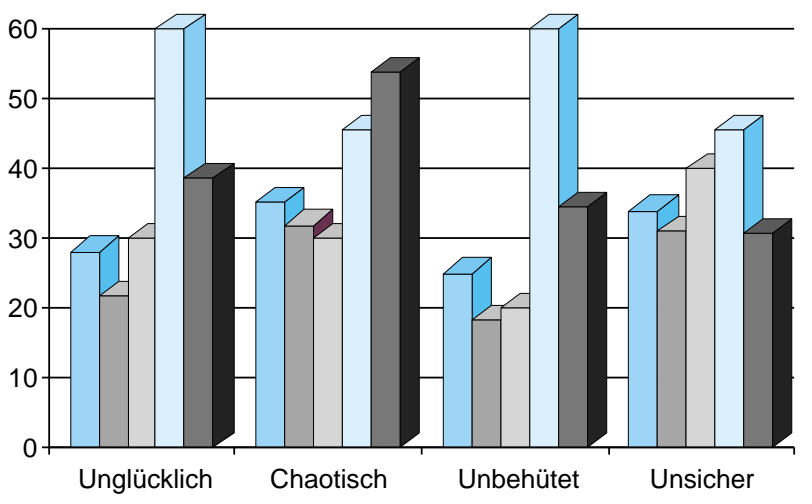

$\square$ Alle

$\square$ Mit suchtkrankem Vater

$\square$ Mit suchtkranker Mutter

$\square$ Mit zwei suchtkranken Eltern

$\square$ Mit Eltern ohne dauerhafte

Abstinenz
Abb. 1 Einschätzung der Kindheit (bis 12. Lebensjahr).
Abb. 2 Einschätzung der Jugend (12. bis 18. Lebensjahr).

\section{Gewalterfahrungen in der Herkunftsfamilie}

Neben der familiären Atmosphäre in der Herkunftsfamilie wurden die ehemaligen Seminarteilnehmer auch nach Gewalterfahrungen befragt. Tab. 3 zeigt die Angaben zu den körperlichen (physischen) Gewalterfahrungen in der Herkunftsfamilie:

Physische Gewalt tritt insgesamt häufiger auf, wenn der Vater oder beide Elternteile suchtkrank sind. Dies bestätigt klar die bereits unter der Fragestellung der Familienatmosphäre gefundenen Ergebnisse. Tägliche oder fast tägliche Gewalt (wie z.B. Prügel, Schläge, Tritte usw.) berichten insgesamt 3,1\% aller Befragten, aber 5,5\% aller Befragten mit einem alkoholabhängigen Vater. Nimmt man die Personen hinzu, die zumindest häufig physische Gewalt in der Familie erlebt haben (oft: mehr als 5-mal monatlich), so ergeben sich folgende Quoten: Die Gesamtgruppe ( $n=98$ ) berichtet in 9,2\% aller Fälle von täglicher oder häufiger physischer Gewalt in der Herkunftsfamilie, diejenigen mit einem suchtkranken Vater zu 14,6\% und diejenigen mit zwei suchtkranken Elternteilen zu 9,1\%. Interessanterweise wird im Falle ausschließlicher mütterlicher Abhängigkeit in keinem einzigen Fall von derartig häufiger körperlicher Gewalt berichtet $(0,0 \%$ !).

Ein etwas anderes Bild ergibt sich hinsichtlich seelischer Gewalterfahrungen (z. B. Drohen, Brüllen, Entwerten) in der Herkunftsfamilie. Hier berichten immerhin 18,3\% aller Befragten von täglichen bzw. fast täglichen Erfahrungen psychischer Gewalt, wie z. B. Schreien, Drohen oder Liebesentzug. Bei Hinzunahme der Kategorie mit der zweithöchsten Frequenz von Gewalterfahrungen (oft: bis zu 5-mal im Monat) steigert sich die Quote auf 35,6\%. Dies spricht für ein hohes intrafamiliales Konflikt- und
Spannungsniveau, wie dies auch schon bei der Auswertung der familienatmosphärischen Daten deutlich wurde. Für diese Variable zeigt sich unter Betrachtung der geschlechtsspezifischen Eltern-Abhängigkeitsbelastung (Vater vs. Mutter vs. beide) ein anderes Bild als bei der physischen Gewalt: Suchtkranke Mütter weisen bei der täglichen psychischen Gewalt eine höhere Quote auf als nicht suchtkranke Mütter, was sich jedoch bei der Kategorie „oft“ deutlich ins Gegenteil zu Lasten der suchtkranken Väter verkehrt (25,5\% im Vergleich zu 4,8\%). Die am stärksten belasteten Gruppen sind jedoch wiederum die mit zwei suchtkranken Elternteilen und mit einem Elternteil, der nach der Therapie keine dauerhafte Abstinenz erreicht hat. So erfahren hier 9,1\% bzw. 7,8\% aller Probanden niemals psychische Gewalt, im Unterschied zu immerhin 21,4\% der Gesamtgruppe.

\section{Bewertung der Eltern und der eigenen Kindheit und Jugend}

In den folgenden Tab. $\mathbf{4}$ und $\mathbf{5}$ sind die Ergebnisse der Einschätzung von Vater und Mutter während Kindheit bzw. Jugend bezüglich wichtiger Erlebensbereiche wiedergegeben. Die Probanden wurden mittels bipolarer sechsstufiger Items befragt, wie sie Mutter bzw. Vater in ihrer Kindheit und Jugend erlebt hatten. In den beiden Tabellen sind jeweils die positiven Pole der Items (wichtig - unwichtig; liebenswert - nicht liebenswert; positiv negativ; verständnisvoll - verständnislos) wiedergegeben. Inhaltlich zeigt sich, dass die Mütter von der Gesamtgruppe der Befragten günstiger eingeschätzt wurden als die Väter. Dies mag zum einen damit zusammenhängen, dass Mütter in der Erziehung der Kinder eine wichtigere und entscheidendere Rolle einnehmen als Väter, zum anderen aber auch an der Tatsache liegen, dass mehr Väter als Mütter der Stichprobe suchtkrank waren. Im Einzelnen wurden die Mütter im Unterschied zu den Vätern zu 
Tab. 4 Wie erlebten Sie während Ihrer Kindheit und Jugend Ihre Mutter? (alle Angaben in Prozent)

\begin{tabular}{|c|c|c|c|c|c|}
\hline Kategorie & $\begin{array}{l}\text { alle } \\
n=98\end{array}$ & $\begin{array}{l}\text { mit } \\
\text { suchtkrankem } \\
\text { Vater } \\
n=61\end{array}$ & $\begin{array}{l}\text { mit } \\
\text { suchtkranker } \\
\text { Mutter } \\
n=24\end{array}$ & $\begin{array}{l}\text { mit zwei } \\
\text { suchtkranken } \\
\text { Elternteilen } \\
n=13\end{array}$ & $\begin{array}{l}\text { mit Eltern } \\
\text { ohne dauerhafte } \\
\text { Abstinenz } \\
n=26\end{array}$ \\
\hline wichtig & 89,6 & 96,3 & 71,4 & 80 & 88,5 \\
\hline liebenswert & 88,4 & 94,5 & 65 & 88,9 & 96,2 \\
\hline positiv & 76,6 & 85,2 & 57,1 & 50 & 79,2 \\
\hline verständnisvoll & 78,9 & 90,7 & 60 & 50 & 80 \\
\hline
\end{tabular}

Tab. 5 Wie erlebten Sie während Ihrer Kindheit und Jugend Ihren Vater? (alle Angaben in Prozent)

\begin{tabular}{|c|c|c|c|c|c|}
\hline Kategorie & $\begin{array}{l}\text { alle } \\
n=98\end{array}$ & $\begin{array}{l}\text { mit } \\
\text { suchtkrankem } \\
\text { Vater } \\
n=61\end{array}$ & $\begin{array}{l}\text { mit } \\
\text { suchtkranker } \\
\text { Mutter } \\
n=24\end{array}$ & $\begin{array}{l}\text { mit zwei } \\
\text { suchtkranken } \\
\text { Elternteilen } \\
n=13\end{array}$ & $\begin{array}{l}\text { mit Eltern } \\
\text { ohne dauerhafte } \\
\text { Abstinenz } \\
n=26\end{array}$ \\
\hline wichtig & 73,7 & 70,9 & 85,7 & 62,5 & 68 \\
\hline liebenswert & 64,6 & 63,6 & 81 & 44,4 & 52 \\
\hline
\end{tabular}

89,6\% im Vergleich zu 73,7\% als wichtig, zu 88,4\% im Vergleich zu $64,6 \%$ als liebenswert, zu 76,6\% im Vergleich zu 62,4\% als positiv und zu 78,9\% im Vergleich zu 52,7\% als verständnisvoll eingeschätzt.

Wenn die Untergruppe mit ausschließlich suchtkranken Vätern betrachtet wird, erhöhen sich die Werte für die Mütter um 6\% bis knapp $12 \%$ für die einzelnen Kategorien, während sich die Einschätzung der Väter noch weiter verschlechtert. So werden nur noch etwas mehr als die Hälfte (55,6\%) aller Väter als positiv eingeschätzt und sogar nur noch $47,3 \%$ als verständnisvoll. Interessanterweise sind es aber immer noch $70,9 \%$ aller Väter, die als wichtig eingestuft werden.

Die Untergruppe mit ausschließlich suchtkranken Müttern imponiert mit Vätern, die für alle Kategorien bessere Werte als die Mütter aufweisen. So werden die nicht suchtkranken Väter im Unterschied zu den suchtkranken Müttern zu 85,7\% im Vergleich zu 71,4\% als wichtig, zu $81 \%$ im Vergleich zu $65 \%$ als liebenswert, zu $85 \%$ im Vergleich zu 57,1\% als positiv und zu $75 \%$ im Vergleich zu 60\% als verständnisvoll eingeschätzt. Offenbar gelingt es einem Großteil der jeweils nicht suchtkranken Elternteile in der Beziehungsqualität zu ihren Kindern, die erlebten Defizite im Verhältnis zu den jeweils suchtkranken Elternteilen auszugleichen.

Bei den beiden weiteren Untergruppen, den Probanden mit zwei suchtkranken Elternteilen und mit Eltern ohne dauerhaften Therapieerfolg, zeigen sich differenziell interessante Effekte: Während die Mütter sich in der Beurteilung kaum von den Müttern der anderen Gruppen unterscheiden (ungünstige Ausnahme: „positiv“ und „verständnisvoll“ in der Subgruppe mit zwei sucht- kranken Elternteilen), weichen die Werte für die Väter für alle Kategorien deutlich nach unten ab. So werden nur noch $44,4 \%$ der Väter in der Gruppe mit zwei suchtkranken Elternteilen als liebenswert und 42,9\% als verständnisvoll bewertet. Bei den Eltern ohne dauerhaften Therapieerfolg sind die entsprechenden Werte mit $41,7 \%$ bzw. $36 \%$ sogar noch geringer.

Die Einschätzung der Eltern während der Kindheit und Jugend der Probanden ist zwar nur ein globaler und wenig differenzierter Indikator für eine Vielzahl von Interaktionserfahrungen. Die eruierten Zahlen machen jedoch schon zahlreiche Tendenzen in Richtung problematischer intrafamilialer Beziehungen deutlich. Diese Belastungen nehmen erwartungsgemäß zu, wenn beide Elternteile suchtkrank sind (hier jedoch vornehmlich bezüglich der Väter) und wenn ein Elternteil seine Suchtkrankheit nicht dauerhaft überwindet.

Auf einer differenzierteren Ebene liegt die getrennte Einschätzung der Kindheit (Lebenszeit bis zum Alter von 12 Jahren) und der Jugend (zwischen 12 und 18 Jahren), die bei den Probanden anschließend erfragt wurde. Dafür wurden wiederum vier bipolar konstruierte, sechsstufig skalierte Attribute verwendet: unglücklich - glücklich, chaotisch - geordnet, unbehütet - behütet und unsicher - sicher. Somit konnte jeder Befragte für die ersten 18 Jahre seiner Lebenszeit acht Urteile abgeben. Während von allen 98 Probanden ca. ein Fünftel (je nach Beurteilungsreiz zwischen $17,5 \%$ für unbehütet und $23,7 \%$ für chaotisch bzw. unsicher) ihre Kindheit überwiegend negativ beurteilten, ist diese Quote im Falle väterlicher Abhängigkeit um durchschnittlich 6,1\% geringer, während sie im Falle mütterlicher Abhängigkeit um durchschnittlich 7,9\% erhöht ist. Die sich dadurch ergebende Spannweite von $14 \%$ zwischen den durchschnittlichen Beurtei- 
lungswerten für die Kindheit bei väterlicher bzw. mütterlicher Abhängigkeit macht die signifikante Rolle der Mutter als Hauptbezugsperson im Leben eines Kindes sehr deutlich. Für einzelne Werte ergeben sich noch Steigerungen, wenn wiederum die beiden restlichen Subgruppen, jene mit zwei abhängigen Elternteilen und mit Eltern, die nach der Therapie ohne dauerhafte Abstinenz bleiben, betrachtet werden. Bei der Gruppe mit zwei abhängigen Elternteilen sind es immerhin jeweils 36,4\% der Antworter, die ihre Kindheit als unglücklich und unsicher einstufen. Bei den Probanden, deren Eltern weiterhin abhängig waren, sind es $34,6 \%$, die ihre Kindheit als chaotisch einstufen.

Wird nun im Unterschied zur Kindheit das Jugendalter betrachtet, nach unserer Definition die Altersspanne zwischen 12 und 18 Jahren, ergeben sich fast durchweg höhere Werte. Etwas mehr als durchschnittlich 30\% der Einschätzungen fallen bei der Gesamtgruppe nunmehr negativ aus. Dabei überwiegen die Elemente „Chaos“ (35\%) und „Unsicherheit“ (34\%). Wiederum verringern sich die Werte bei der Subgruppe mit suchtkrankem Vater, während sie sich für die Gruppe mit einer suchtkranken Mutter nicht mehr durchweg erhöhen. Dennoch ist es bemerkenswert, dass 40\% der Probanden mit einer suchtkranken Mutter ihre Jugend als unsicher erlebten. Noch deutlicher wird die „instabile“ Jugend bei den beiden restlichen Subgruppen: Jeweils $60 \%$ der Antworter mit zwei suchtkranken Elternteilen erlebten ihre Jugend als unglücklich bzw. unbehütet. Bei der Gruppe mit Eltern ohne dauerhaften Therapieerfolg sind es nunmehr 53,8\%, die ihre Jugendzeit als chaotisch beurteilen.

\section{Derzeitige Lebenssituation und Problembereiche}

$58(59,2 \%)$ leben derzeit mit ihrer leiblichen Mutter in einem Haushalt, 51 (52\%) mit ihrem leiblichen Vater. Insgesamt leben $61(62,2 \%)$ mit mindestens einem leiblichen Elternteil zusam- men in einem Haushalt. In 8 (8,2\%) Fällen leben die Probanden mit ihren Großeltern in einem Haushalt. 39 (39,8\%) Personen leben in einem Haushalt, in dem auch Geschwister sind. Eigene Kinder, mit denen sie zusammenleben, haben schon 7 (7,1\%) ehemalige Seminarteilnehmer. 21 (21,4\%) der Befragten leben mit einem festen Partner zusammen. 11 (11,2\%) der Antworter leben alleine.

Auf die Frage, welche Verhaltensweisen sie in ihrem heutigen Leben als problematisch erleben, benannten die 98 Probanden im Durchschnitt 2,29 von 15 Bereichen. Wie Tab. 6 außerdem zeigt, sind es bei der Gruppe der Probanden mit suchtkranken Müttern 2,71, bei der mit zwei suchtkranken Elternteilen 2,63 und bei der mit Eltern, die keine dauerhafte Abstinenz erreichten, sogar 3,38 Problembereiche. Im Einzelnen sind es Stimmungsschwankungen (40,8\%), die von der Gesamtgruppe am häufigsten als problematisch genannt werden. Am zweithäufigsten ist der Mangel an Selbstwertgefühlen (30,6\%) benannt. Bei den klinisch psychologisch als schwerwiegender zu beurteilenden Symptomen stechen Ängste (25,5\%), Depressionen (21,4\%), problematisches Essverhalten (19,4\%) und Zwänge (18,4\%) hervor. Insgesamt zeigt sich, dass ein Fünftel bis ein Viertel der Untersuchungsgruppe nach den Selbstauskünften als psychisch belastet anzusehen ist. Problematischen Alkoholkonsum berichten 6,1\%, problematischen Drogenkonsum 3,1\% der Probanden. Einen problematischen Medikamentenkonsum berichten weniger als $1 \%$ der Antworter, weshalb er in Tab. 6 auch nicht aufgenommen wurde. Werden wiederum die Subgruppen betrachtet, so ergeben sich für die Gruppe mit einer suchtkranken Mutter auf einigen der Problembereiche erhöhte Werte: Problematischer Alkoholkonsum wird von 9,5\%, problematischer Arbeitsstil von 23,8\% und ein starker Mangel an Selbstwertgefühl von $42,9 \%$ berichtet. Bei der Gruppe mit zwei suchtkranken Elternteilen sind die Wer-

Tab. 6 Aktuell problematische Lebensbereiche im Selbsturteil junger erwachsener Kinder aus suchtbelasteten Familien (alle Angaben in Prozent)

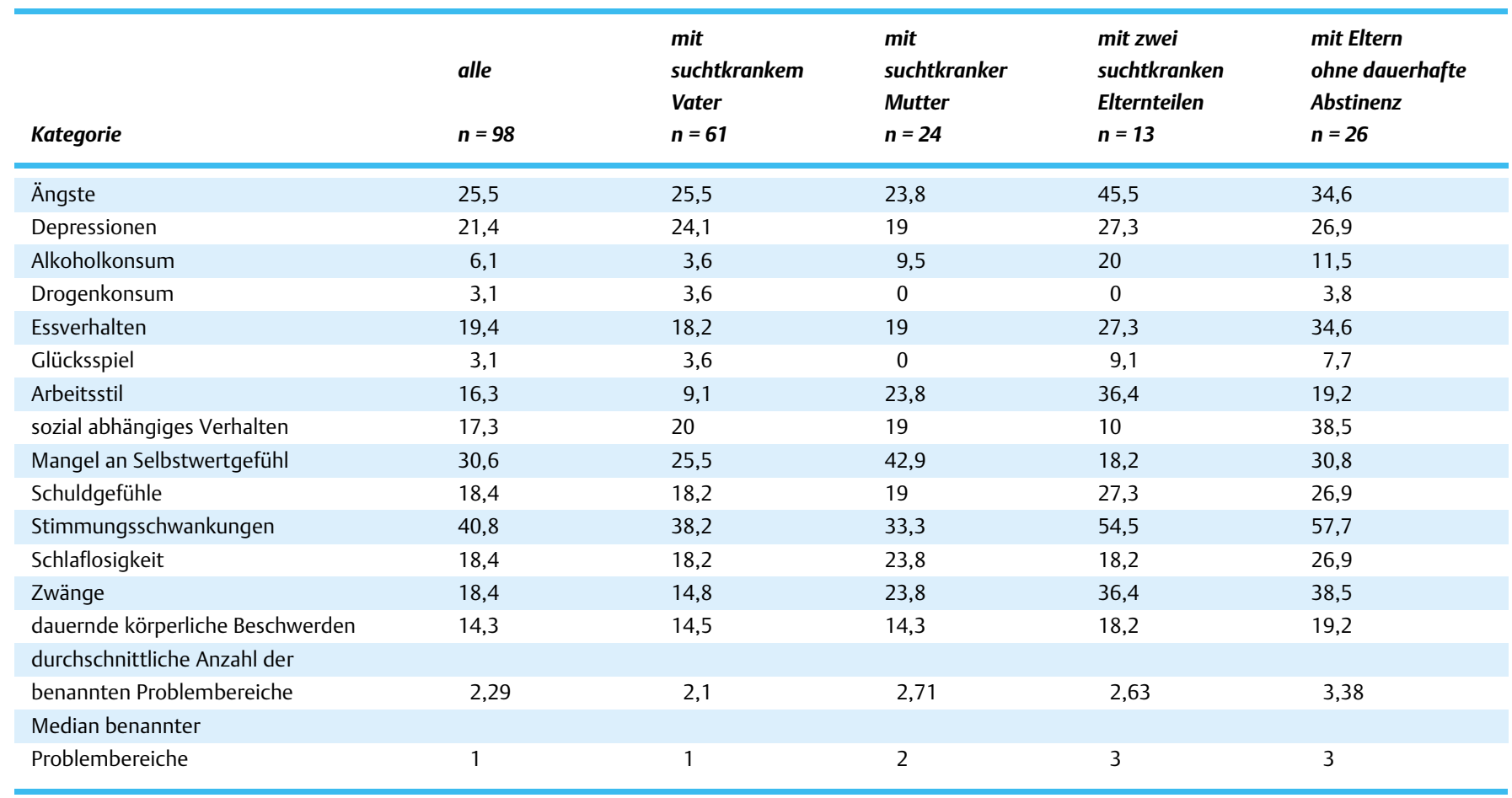


te für Ängste (45,5\%), problematischen Alkoholkonsum (20\%), problematisches Essverhalten (27,3\%), problematischen Arbeitsstil (36,4\%), Schuldgefühle (27,3\%), Stimmungsschwankungen $(54,5 \%)$ und Zwänge $(36,4 \%)$ deutlich erhöht. Erneut zeigt sich diese Gruppe als eine besonders stark belastete Subgruppe erwachsener Kinder aus suchtbelasteten Familien. Es ist jedoch auch hier hervorzuheben, dass ein zahlenmäßig nicht unbedeutender Teil von ihnen keine Problembelastungen benennt. Die Gruppe der Probanden mit Eltern ohne dauerhaften Therapieerfolg ist besonders in den Bereichen Ängste (34,6\%), problematisches Essverhalten (34,6\%), sozial abhängiges Verhalten (38,5\%), Stimmungsschwankungen (57,7\%) und Zwänge (38,5\%) belastet. Sie weisen außerdem - wie schon angegeben - den höchsten Median berichteter Problembereiche auf, so dass bei ihnen die stärkste individuelle Multiproblembelastung vorliegen dürfte.

\section{Diskussion}

Die vorliegende Untersuchung beschäftigte sich mit der Langzeitentwicklung junger erwachsener Kinder aus suchtbelasteten Familien, wobei ein Elternteil während der Kindheit oder Jugend der Befragten eine stationäre Entwöhnungsbehandlung absolvierte.

Erfreulicherweise zeigte sich in unserer fremdanamnestischen Katamnese, dass in nur 26 der 98 Fälle (26,5\%), die antworteten, der suchtkranke Elternteil keine dauerhafte Abstinenz erreicht hatte. Auch wenn es sich um eine hochselektive Klientel handelt, ist der zu verzeichnende Erfolgswert von drei Vierteln (73,5\%) dauerhafter Abstinenz bei einer durchschnittlichen Katamnesedauer von 8 Jahren als sehr gut einzuschätzen. Werden die Eltern der nicht antwortenden ehemaligen Seminarteilnehmer nach konservativem Vorgehen als rückfällig eingestuft, ergibt sich eine vermutete Abstinenzquote für die gesamte Untersuchungsgruppe von 37,1\% (72 von 194 ehemaligen Patienten). Es ist jedoch mit Sicherheit davon auszugehen, dass dieser Wert den wahren Wert unterschätzt.

Die Tatsache, dass nicht alle Befragten als Kinder unter der Suchterkrankung ihrer Eltern erkennbar litten und in der aktuellen Situation manifeste Störungen aufweisen, ist weiterhin bemerkenswert und deckt sich mit den Ergebnissen der neueren Resilienzforschung [13]. Dies unterstreicht die oft unterschätzte Heterogenität innerhalb der Gruppe der Kinder von Alkoholikern und macht die Notwendigkeit differenzieller Diagnose- und Präventionsstrategien deutlich [vgl. 1].

Unter differenzieller Betrachtung zeigen sich ungünstige Werte vor allem dann, wenn beide Elternteile alkoholabhängig sind oder wenn bei dem behandelten suchtkranken Elternteil keine dauerhafte Abstinenz eintritt. Für manche Variablen ergeben sich ungünstigere Werte, wenn die Mütter suchtkrank sind. Dies unterstreicht die herausragende Rolle der Mutter-Kind-Beziehung für die seelische Gesundheit der heranwachsenden Kinder. Allerdings spielen auch väterliche Verhaltensweisen (z.B. physische Gewalt) eine deutlich beeinträchtigende Rolle für die Entwicklung der Kinder.
In ihrer aktuellen Lebenssituation zeigen sich etwa $20 \%$ bis $25 \%$ der jungen erwachsenen Kinder aus Suchtfamilien beeinträchtigt. Es bestätigt sich hierin die wiederholt gefundene stärkere Belastung dieser Gruppe. Besonders hervorzuheben sind die Belastungen der Probanden in den zentralen psychischen Störungsbereichen Angst und Depression. Lachner u. Wittchen [2] berichten in Übereinstimmung mit unseren Ergebnissen, dass knapp $30 \%$ der Kinder von Eltern mit einer alkoholbezogenen Diagnose im Alter zwischen 14 und 24 Jahren eine Angststörung, 24,8\% eine Phobie, und $13 \%$ eine affektive Störung aufweisen. Dies verdeutlicht die Notwendigkeit, gerade bei frühen neurotischen Störungen von Kindern und Jugendlichen auf einen eventuell vorhandenen familiären Lern- und Erfahrungshintergrund im Bereich von Sucht und Abhängigkeitserkrankungen zu achten.

Ob die „wahre“ Belastung bei der Gesamtheit der Kinder aus suchtbelasteten Familien noch höher liegt, was durch die Selektivität der Antwortergruppe nahe gelegt wird, muss zunächst offen bleiben. Auch das Fehlen von Vergleichsgruppen Jungerwachsener, die in normalen familiären oder anderweitig dysfunktionalen Kontexten aufgewachsen sind, schränkt die Generalisierbarkeit der Ergebnisse ein. Solche Studien werden derzeit durchgeführt, um hier weiteren Aufschluss zu bringen.

\section{Literatur}

${ }^{1}$ Klein M, Zobel M. Kinder aus alkoholbelasteten Familien. Kindheit und Entwicklung. Zeitschrift für Klinische Kinderpsychologie 1997; 6: $133-140$

${ }^{2}$ Lachner G, Wittchen HU. Familiär übertragende Vulnerabilitätsmerkmale für Alkoholmissbrauch und -abhängigkeit. In: Watzl H, Rockstroh B (Hrsg). Abhängigkeit und Missbrauch von Alkohol und Drogen. Göttingen: Hogrefe 1997; 43-89

${ }^{3}$ McKenna T, Pickens R. Alcoholic children of alcoholics. Journal of Studies on Alcohol 1981; 42: 1021 - 1029

${ }^{4}$ Hesselbrock VM, Stabenau JR, Hesselbrock MN et al. The nature of alcoholism in patients with different familiy histories for alcoholism. Progress in Neuro-Psychopharmacology and Biological Psychiatry 1982; 6: 607-614

${ }^{5}$ Zobel M, Rösch W, Quinten C. Jahresbericht 1993. Zahlen - Daten Fakten. Thommener Therapie Texte 1994; 6 (Heft 2)

${ }^{6}$ Cotton NS. The familial incidence of alcoholism. Journal of Studies on Alcohol 1979; 40: 89-116

${ }^{7}$ Drake RE, Vaillant GE. Predicting alcoholism and personality disorder in a 33-year longitudinal study of children of alcoholics. British Journal of Addiction 1988; 83: 799-807

${ }^{8}$ Schuckit MA. Low level response to alcohol as a predictor of future alcoholism. American Journal of Psychiatry 1994; 151: 184- 189

${ }^{9}$ Schuckit MA, Smith TL. An 8-year follow-up of 450 sons of alcoholic and control subjects. Archives of General Psychiatry 1996; 53: $202-210$

${ }^{10}$ Sigvardsson S, Bohman M, Cloninger R. Replication of the Stockholm Adoption Study of Alcoholism. Confirmatory Cross-Fostering Analysis. Archives of General Psychiatry 1996; 53: 681-687

${ }^{11}$ Sher KJ. Children of alcoholics. A critical appraisal of theory and research. Chicago: University of Chicago Press 1991

12 Velleman R. Intergenerational effects - a review of environmentally oriented studies concerning the relationship between parental aclohol problems and family disharmony in the genesis of alcohol and other problems. II. The intergenerational effects of family disharmony. The International Journal of the Addictions 1992; 27: 367-389

${ }^{13}$ Wolin S, Wolin S. Resilience among youth growing up in substanceabusing families. Substance Abuse 1995; 42: 415-429 